ELECTRONIC RESEARCH ANNOUNCEMENTS OF THE AMERICAN MATHEMATICAL SOCIETY

Volume 5, Pages 40-46 (April 6, 1999)

S $1079-6762(99) 00060-8$

\title{
ON BOJARSKI'S INDEX FORMULA FOR NONSMOOTH INTERFACES
}

\author{
MARIUS MITREA
}

(Communicated by Stuart Antman)

\begin{abstract}
Let $D$ be a Dirac type operator on a compact manifold $\mathcal{M}$ and let $\Sigma$ be a Lipschitz submanifold of codimension one partitioning $\mathcal{M}$ into two Lipschitz domains $\Omega_{ \pm}$. Also, let $\mathcal{H}_{+}^{p}(\Sigma, D)$ be the traces on $\Sigma$ of the $\left(L^{p_{-}}\right.$ style) Hardy spaces associated with $D$ in $\Omega_{ \pm}$. Then $\left(\mathcal{H}_{-}^{p}(\Sigma, D), \mathcal{H}_{+}^{p}(\Sigma, D)\right)$ is a Fredholm pair of subspaces for $L^{p}(\Sigma)$ (in Kato's sense) whose index is the same as the index of the Dirac operator $D$ considered on the whole manifold $\mathcal{M}$.
\end{abstract}

\section{INTRODUCTION AND STATEMENT OF RESULTS}

Let $\Sigma$ be a rectifiable curve in $\mathbb{R}^{2} \equiv \mathbb{C}$ which divides the plane into two domains, denoted by $\Omega_{ \pm}$. Corresponding to these, introduce the Hardy spaces on $\Sigma$,

$$
\mathcal{H}_{ \pm}^{p}(\Sigma):=\left\{\left.u\right|_{\Sigma} ; \bar{\partial} u=0 \text { in } \Omega_{ \pm}, \mathcal{N}(u) \in L^{p}(\Sigma)\right\}, \quad 1<p<\infty,
$$

where $\bar{\partial}$ is the usual Cauchy-Riemann operator and $\mathcal{N}$ is the so-called nontangential maximal operator. More concretely, for $x \in \Sigma, \mathcal{N}(u)(x):=\sup \left\{|u(y)| ; y \in \gamma_{ \pm}(x)\right\}$, where $\gamma_{ \pm}(x) \subseteq \Omega_{ \pm}$are suitable nontangential approach regions; cf., e.g., [Ke]. A classical result in complex and harmonic analysis is the Plemelj-Calderón decomposition

$$
L^{p}(\Sigma)=\mathcal{H}_{-}^{p}(\Sigma) \oplus \mathcal{H}_{+}^{p}(\Sigma), \quad 1<p<\infty,
$$

valid under suitable assumptions on $\Sigma$. Among other things, (1.2) is equivalent to $\Sigma$ being Ahlfors regular and to the $L^{p}$-boundedness of the (principal value) Cauchy integral operator on $\Sigma$. See [Da] and [MeCo] for a more detailed discussion in this regard.

The decomposition (1.2) can also be interpreted as a transmission problem for $\bar{\partial}$ and, as such, has intimate connections with the classical Riemann-Hilbert problem in the plane. See $[\mathrm{Mu}]$ for a treatment from the classical point of view of the latter. Extensions of (1.2) to the higher dimensional setting can be done in the Clifford algebra setting. In this case, $\bar{\partial}$ is replaced by a Dirac operator and $\Sigma$ can be taken to be a Lipschitz hypersurface in $\mathbb{R}^{m}$. This has been worked out in [LMcS], [GM], [Mi].

Let $\mathcal{M}$ be a smooth, connected, compact, Riemannian manifold without boundary, and let $\mathcal{E}, \mathcal{F} \rightarrow \mathcal{M}$ be two smooth Hermitian vector bundles. We assume

Received by the editors December 02, 1998.

1991 Mathematics Subject Classification. Primary 58G10, 42B20; Secondary 34L40, 30D55.

Partially supported by NSF.

(C)1999 American Mathematical Society 
that all metric structures have coefficients in the Sobolev space $H^{2, r}$ for some $r>m:=\operatorname{dim} \mathcal{M}$. Our aim is to investigate the analogue of $(1.2)$ when $\Sigma$ is a Lipschitz submanifold of codimension one of $\mathcal{M}$ and $D: \mathcal{E} \rightarrow \mathcal{F}$ is a first-order, elliptic differential operator whose coefficients have a limited amount of smoothness. Specifically, we assume that the top coefficients of $D$ belong to $H^{2, r}$,

for some $r>m$. Under the current assumptions, $D^{*}$, the adjoint of $D$, also satisfies (1.3)-(1.4).

The major analytical hypothesis we make on the differential operator is that

$$
D \text { and } D^{*} \text { have the unique continuation property. }
$$

Recall that $D$ is said to have the unique continuation property if the following holds: $u \in H^{1,2}(\mathcal{M}, \mathcal{E})$ and $D u=0$ implies that either $u \equiv 0$ or $\operatorname{supp} u=\mathcal{M}$. The hypothesis (1.5) is natural inasmuch as it is automatically satisfied (for $r=\infty$ ) by Dirac type operators; cf. [Ar], [Co].

Consider now a Lipschitz subdomain $\Omega$ of $\mathcal{M}$ and set $\Sigma:=\partial \Omega, \Omega_{+}:=\Omega$, $\Omega_{-}:=\mathcal{M} \backslash \bar{\Omega}$. Also, for $1<p<\infty$, introduce the Hardy type spaces

$$
\mathcal{H}^{p}\left(\Omega_{ \pm}, D\right):=\left\{u: \Omega_{ \pm} \rightarrow \mathcal{E} ; D u=0 \text { in } \Omega_{ \pm}, \mathcal{N}(u) \in L^{p}(\Sigma)\right\},
$$

and

$$
\mathcal{H}_{ \pm}^{p}(\Sigma, D):=\left\{\left.u\right|_{\Sigma} ; u \in \mathcal{H}^{p}\left(\Omega_{ \pm}, D\right)\right\} .
$$

Here, the trace $\left.u\right|_{\Sigma}$ is considered in the nontangential pointwise sense, i.e. for $x \in \Sigma$, $\left.u\right|_{\Sigma}(x)=\lim u(y)$ as $y \rightarrow x$ and $y \in \gamma_{ \pm}(x)$. That this makes sense a.e. on $\Sigma$ will follow a posteriori from our main result. Preparatory to stating this theorem we need one more definition. Recall from $[\mathrm{Ka}]$ that $(A, B)$ is called a Fredholm pair for the Banach space $X$ if $A, B$ are closed subspaces of $X$ so that

$$
\operatorname{dim}(A \cap B)<\infty, \quad \operatorname{dim}(X /(A+B))<\infty .
$$

In this case, one defines Index $(A, B):=\operatorname{dim}(A \cap B)-\operatorname{dim}(X /(A+B))$. Finally, recall the smoothness index $r$ from (1.3)-(1.4).

Theorem 1.1. Let the vector bundles $\mathcal{E}, \mathcal{F} \rightarrow \mathcal{M}$, and the differential operator $D: \mathcal{E} \rightarrow \mathcal{F}$ satisfy the above hypotheses. Then, for any Lipschitz submanifold $\Sigma$ of codimension one in $\mathcal{M}$ (arising as the boundary of a Lipschitz subdomain of $\mathcal{M}$ ), the Hardy spaces $\left(\mathcal{H}_{-}^{p}(\Sigma, D), \mathcal{H}_{+}^{p}(\Sigma, D)\right)$ are a Fredholm pair for $L^{p}(\Sigma, \mathcal{E})$ and

$$
\operatorname{Index}\left(\mathcal{H}_{-}^{p}(\Sigma, D), \mathcal{H}_{+}^{p}(\Sigma, D)\right)=\operatorname{Index}\left(D: H^{1, p}(\mathcal{M}, \mathcal{E}) \rightarrow L^{p}(\mathcal{M}, \mathcal{F})\right)
$$

for each $1<p<\infty$. Moreover, for the same range of $p$ 's,

$$
\begin{aligned}
& L^{p}(\Sigma, \mathcal{E})=\mathcal{H}_{-}^{p}(\Sigma, D) \oplus \mathcal{H}_{+}^{p}(\Sigma, D) \\
& \quad \Leftrightarrow D: H^{1, p}(\mathcal{M}, \mathcal{E}) \rightarrow L^{p}(\mathcal{M}, \mathcal{F}) \text { is an isomorphism. }
\end{aligned}
$$

Thus, in the present context, the analogue of (1.2) is valid only modulo finite dimensional linear spaces. Note that while $\mathcal{H}_{ \pm}^{p}(\Sigma, D)$ live on (the lower dimensional manifold) $\Sigma$, they originate from complementary "semi-global" data on $\mathcal{M}$.

While Theorem 1.1 can be naturally seen as a statement about a transmission boundary problem for $D$ across the interface $\Sigma$, there are also clear connections with more classical aspects of index theory. In fact, a version of Theorem 1.1 
corresponding to $p=2$ in the smooth case has been first conjectured by B. Bojarski in mid 1970's (cf. [Bo]) in an effort to produce a surgery type proof of the celebrated Atyah-Singer index theorem. Subsequently, Bojarski's conjecture has been proved by B. Booß-Bavnbek and K. Wojciechowski in mid 1980's ([BBW1]). Other recent extensions (involving the spectral flow and the Maslov index) can be found in $[\mathrm{Ni}]$; see also the broader account in [BBW2].

The primary interest in Theorem 1.1 stems from the low regularity assumptions we make on the metrics, the coefficients of $D$ and the interface $\Sigma$. As is well known in $\mathrm{PDE}$, the character of the problem at hand changes once irregularities are allowed. Among other things, the approach in [BBW1], [BBW2] makes substantial use of the symbolical calculus for pseudodifferential operators and, hence, does not readily extend to the nonsmooth case we consider here. Our solution, mimicking complexvariable type arguments, uses harmonic analysis tools such as Calderón-Zygmund theory for singular integral operators and nontangential function estimates. Some of the basic steps are sketched below.

\section{ACKNOWLEDGMENTS}

I thank B. Booß-Bavnbek for several insightful discussions.

\section{Outline OF PROOF}

In this section we indicate the main steps involved in the proof of Theorem 1.1; the missing details will appear elsewhere.

Step I. Fix $V \in C^{\infty}(\mathcal{M})$, a scalar-valued, positive, non-identically zero function with $\bar{\Omega} \cap \operatorname{supp} V=\emptyset$ and consider the second-order, formally self-adjoint differential operator $\tilde{L}:=D^{*} D+V$. Then, invoking $(1.5)$, it follows that $\tilde{L}: H^{1,2}(\mathcal{M}, \mathcal{E}) \rightarrow$ $H^{-1,2}(\mathcal{M}, \mathcal{E})$ is invertible. Consider $\tilde{E} \in \mathcal{D}^{\prime}(\mathcal{M} \times \mathcal{M}, \mathcal{E} \otimes \mathcal{E})$ the Schwartz kernel of $\tilde{L}^{-1}$ and set

$$
\tilde{\Gamma}(x, y):=\left(\operatorname{Id}_{x} \otimes \bar{D}_{y}\right) \tilde{E}(x, y), \quad \tilde{\Gamma} \in \mathcal{D}^{\prime}(\mathcal{M} \times \mathcal{M}, \mathcal{E} \otimes \mathcal{F}),
$$

where $\bar{D} u:=\left[D u^{c}\right]^{c}$ and $[\ldots]^{c}$ denotes complex conjugation. Next, for arbitrary sections $f: \partial \Omega \rightarrow \mathcal{E}$ we introduce the Cauchy type operator

$$
\tilde{\mathcal{C}} f(x):=\int_{\partial \Omega}\langle\tilde{\Gamma}(x, y), i \sigma(D ; \nu(y)) f(y)\rangle_{y} d S(y), \quad \text { for } x \in \Omega .
$$

Here $d S$ stands for the surface measure on $\partial \Omega, \nu \in T^{*} \mathcal{M}$ is the outward unit conormal defined a.e. on $\Sigma=\partial \Omega$, and we denote by $\sigma(P ; \xi)$ the principal symbol of an operator $P$ at $\xi \in T^{*} \mathcal{M}$. Two properties exhibited by this operator and which are of importance for us are as follows. First, for each $1<p<\infty$,

$$
\|\mathcal{N}(\tilde{\mathcal{C}} f)\|_{L^{p}(\partial \Omega)} \leq C\|f\|_{L^{p}(\partial \Omega, \mathcal{E})},
$$

uniformly for $f \in L^{p}(\partial \Omega, \mathcal{E})$. Second,

$$
\text { there exists }\left.\tilde{\mathcal{C}} f\right|_{\partial \Omega} \text { (nontangentially) pointwise a.e. }
$$

for any $f \in L^{p}(\partial \Omega, \mathcal{E}), 1<p<\infty$. These are seen by extending the main results on the Euclidean Cauchy operator on Lipschitz curves in $[\mathrm{CMcM}]$ to the present context (cf. [Mi2]). 
Several notable consequences at the level of Hardy spaces follow more or less directly from these. To begin with, (2.4), integrations by parts and a weak-star argument yield the Cauchy reproducing formula

$$
u=\tilde{\mathcal{C}}\left(\left.u\right|_{\partial \Omega}\right) \quad \text { in } \Omega, \quad \forall u \in \mathcal{H}^{p}(\Omega, D) .
$$

The existence of the nontangential boundary trace $\left.u\right|_{\partial \Omega}$ is a byproduct of the proof of (2.5).

Going further, (2.3) and (2.5) imply the following $L^{p}$-version of the maximum principle:

$$
\|u\|_{L^{p}(\partial \Omega, \mathcal{E})} \approx\|\mathcal{N}(u)\|_{L^{p}(\partial \Omega)}, \quad \text { uniformly for } u \in \mathcal{H}^{p}(\Omega, D) .
$$

As a corollary, $\mathcal{H}_{ \pm}^{p}(\Sigma, D)$ are closed subspaces of $L^{p}(\Sigma, \mathcal{E})$ for any $1<p<\infty$.

Step II. Consider two positive, scalar-valued functions $V_{ \pm} \in C^{\infty}(\mathcal{M})$, non-identically zero and such that $\bar{\Omega}_{ \pm} \cap \operatorname{supp} V_{ \pm}=\emptyset$. Also, set $L_{ \pm}:=D D^{*}+V_{ \pm}$. Once again, from (1.5) it follows that $L_{ \pm}: H^{1,2}(\mathcal{M}, \mathcal{F}) \rightarrow H^{-1,2}(\mathcal{M}, \mathcal{F})$ are invertible, and we denote by $E_{ \pm} \in \mathcal{D}^{\prime}(\mathcal{M} \times \mathcal{M}, \mathcal{F} \otimes \mathcal{F})$ the Schwartz kernels of $L_{ \pm}^{-1}$. Also, introduce $\Gamma_{ \pm}(x, y):=\left(D_{x}^{*} \otimes \operatorname{Id}_{y}\right) E_{ \pm}(x, y), \Gamma_{ \pm} \in \mathcal{D}^{\prime}(\mathcal{M} \times \mathcal{M}, \mathcal{E} \otimes \mathcal{F})$ and the Cauchy type operators acting on arbitrary sections $f: \partial \Omega \rightarrow \mathcal{E}$ by

$$
\mathcal{C}_{ \pm} f(x):=\int_{\partial \Omega}\left\langle\Gamma_{ \pm}(x, y), i \sigma(D ; \nu(y)) f(y)\right\rangle_{y} d S(y), \quad \text { for } x \notin \partial \Omega
$$

and

$$
C_{ \pm} f(x):=\text { p.v. } \int_{\partial \Omega}\left\langle\Gamma_{ \pm}(x, y), i \sigma(D)(y, \nu(y)) f(y)\right\rangle_{y} d S(y), \quad \text { for } x \in \partial \Omega .
$$

Here "p.v." indicates that the integral is taken in the principal value sense, i.e. by removing geodesic balls (with respect to some smooth background Riemannian metric).

There are several properties of these operators which are going to be of importance for us in the sequel. First, $\mathcal{C}_{ \pm}: L^{p}(\partial \Omega, \mathcal{E}) \rightarrow \mathcal{H}^{p}\left(\Omega_{ \pm}, D\right)$ are bounded for each $1<p<\infty$, i.e.

$$
D \mathcal{C}_{ \pm} f=0 \text { in } \Omega_{ \pm} \text {and }\left\|\mathcal{N}\left(\mathcal{C}_{ \pm} f\right)\right\|_{L^{p}(\partial \Omega)} \leq C\|f\|_{L^{p}(\partial \Omega, \mathcal{E})}, \quad \forall f \in L^{p}(\partial \Omega, \mathcal{E})
$$

Second, for all $f \in L^{p}(\partial \Omega, \mathcal{E})$,

$$
\left.\mathcal{C}_{+} f\right|_{\partial \Omega_{ \pm}}=\left( \pm \frac{1}{2} I+C_{+}\right) f,\left.\quad \mathcal{C}_{-} f\right|_{\partial \Omega_{ \pm}}=\left( \pm \frac{1}{2} I+C_{-}\right) f \text { a.e. on } \Sigma,
$$

and, third,

$$
C_{ \pm} \text {are bounded on } L^{p}(\Sigma, \mathcal{E}), \quad 1<p<\infty .
$$

One immediate conclusion is that

$$
\operatorname{Im}\left( \pm \frac{1}{2} I+C_{ \pm} ; L^{p}(\Sigma, \mathcal{E})\right) \subseteq \mathcal{H}_{ \pm}^{p}(\Sigma, D), \quad 1<p<\infty .
$$

Going further, a key observation is that the main singularity in $\Gamma_{ \pm}(x, y)$ is independent of $V_{ \pm}$. In particular, if $m:=\operatorname{dim} \mathcal{M}$, then

$$
\Gamma_{+}(x, y)-\Gamma_{-}(x, y)=O\left(|x-y|^{-(m-1-\epsilon)}\right)
$$

for some $\epsilon>0$. Hence, the integral operator $K:=C_{+}-C_{-}$is compact from $L^{p}(\Sigma, \mathcal{E})$ into itself, $1<p<\infty$. Now, since $I+K=\left(\frac{1}{2} I+C_{+}\right)-\left(-\frac{1}{2} I+C_{-}\right)$, it follows from (2.12) that

$$
\operatorname{Im}\left(I+K ; L^{p}(\Sigma, \mathcal{E})\right) \subseteq \mathcal{H}_{+}^{p}(\Sigma, D)+\mathcal{H}_{-}^{p}(\Sigma, D) .
$$


Consequently, since $I+K$ is Fredholm, it follows that $\mathcal{H}_{+}^{p}(\Sigma, D)+\mathcal{H}_{-}^{p}(\Sigma, D)$ is closed and has finite codimension in $L^{p}(\Sigma, \mathcal{E})$.

Step III. We shall now need the fact that

$$
D: H^{1, p}(\mathcal{M}, \mathcal{E}) \rightarrow L^{p}(\mathcal{M}, \mathcal{F})
$$

is a Fredholm operator for $1<p<\infty$. Furthermore, for $1<p<\infty$,

$$
u \in L^{p}(\mathcal{M}, \mathcal{E}) \text { and } D u=0 \Longrightarrow u \in H^{1, p} \cap C^{\alpha} \text { for some } \alpha>0 .
$$

These are proved by implementing a symbol decomposition to the effect that $D=$ $D^{\#}+D^{b}+B$ with

$$
D^{\#} \in O P C^{\infty} S_{1, \delta}^{1}, \quad D^{b} \in O P C^{1} S_{1, \delta}^{1-\delta}, \quad B \in L^{\infty}(\mathcal{M}, \operatorname{Hom}(\mathcal{E}, \mathcal{F})),
$$

for some $0<\delta<1$. Now (2.14)-(2.15) follow from the existence of a parametrix for $D^{\#}$ and mapping properties for pseudodifferential operators whose symbols have a limited amount of smoothness. See, e.g., [Ta] for references and a general discussion of such issues.

Now, if $f \in \mathcal{H}_{-}^{p}(\Sigma, D) \cap \mathcal{H}_{+}^{p}(\Sigma, D)$, then there exist (unique, by (2.6)) functions $u_{ \pm} \in \mathcal{H}^{p}\left(\Omega_{ \pm}, D\right)$ so that $\left.u_{+}\right|_{\Sigma}=f=\left.u_{-}\right|_{\Sigma}$. Set $u:=u_{-}$in $\Omega_{-}$and $u:=u_{+}$in $\Omega_{+}, u \in L^{p}(\mathcal{M}, \mathcal{E})$ so that $u \in \operatorname{Ker}\left(D: H^{1, p}(\mathcal{M}, \mathcal{E}) \rightarrow L^{p}(\mathcal{M}, \mathcal{F})\right)$. Consequently, the (just defined) assignment $f \mapsto u$ is linear, well defined and, by (2.6), one-toone from $\mathcal{H}_{-}^{p}(\Sigma, D) \cap \mathcal{H}_{+}^{p}(\Sigma, D)$ into $\operatorname{Ker}\left(D: H^{1, p}(\mathcal{M}, \mathcal{E}) \rightarrow L^{p}(\mathcal{M}, \mathcal{F})\right)$. Invoking (2.15), it is clear that this is also onto. Hence,

$$
\operatorname{dim}\left(\mathcal{H}_{-}^{p}(\Sigma, D) \cap \mathcal{H}_{+}^{p}(\Sigma, D)\right)=\operatorname{dim} \operatorname{Ker}\left(D: H^{1, p}(\mathcal{M}, \mathcal{E}) \rightarrow L^{p}(\mathcal{M}, \mathcal{F})\right)<\infty .
$$

At this point, the first part in Theorem 1.1 follows. There remain (1.9) and (1.10), which require refining further the analysis carried out so far. This makes the object of the next couple of steps.

Step IV. Let $[\ldots]^{\circ}$ stand for the annihilator of $[\ldots]$ under the pairing $(u, v)=$ $\int_{\Sigma}\left\langle u, v^{c}\right\rangle d S$. We shall prove that the mapping

$$
\Phi: \mathcal{H}_{ \pm}^{p}\left(\Sigma, D^{*}\right) \rightarrow\left[\mathcal{H}_{ \pm}^{q}(\Sigma, D)\right]^{\circ}, \Phi(u):=i \sigma\left(D^{*} ; \nu\right) u, \quad 1 / p+1 / q=1,
$$

is an isomorphism for any $1<p<\infty$. Note that, in particular, this entails the orthogonal decompositions

$$
L^{2}(\Sigma, \mathcal{E})=\mathcal{H}_{ \pm}^{2}(\Sigma, D) \oplus i \sigma\left(D^{*} ; \nu\right) \mathcal{H}_{ \pm}^{2}\left(\Sigma, D^{*}\right) .
$$

Indeed, $\Phi$ is well defined as an integration by parts shows and is one-to-one because of the ellipticity of $D$. There remains the fact that $\Phi$ is also onto.

We approach this problem as follows. The first observation is that there is no loss of generality in assuming that $\mathcal{E}=\mathcal{F}$ and $D=D^{*}$, the adjoint of $D$. This is seen by "symmetrizing" the operator $D$, i.e. by working with

$$
\mathbb{D}:=\left(\begin{array}{cc}
0 & D \\
D^{*} & 0
\end{array}\right) \quad \text { on } \mathcal{F} \oplus \mathcal{E}
$$

since the original claim can be ultimately recovered from the corresponding one for $\mathbb{D}$. Next, we produce an invertible perturbation $\tilde{D}$ of $D$. More specifically, there exists a symmetric, smoothing operator $P \in L^{-\infty}$ supported away from $\bar{\Omega}$ so that $\tilde{D}:=D-P$ is invertible, say, from $H^{1,2}(\mathcal{M}, \mathcal{E})$ into $L^{2}(\mathcal{M}, \mathcal{E})$. This is done using elementary linear algebra and (1.5). 
Denote by $\Theta(x, y)$ the Schwartz kernel of $\tilde{D}^{-1}$ and introduce the corresponding Cauchy integral operators

$$
\begin{aligned}
& \mathcal{C}_{D} f(x):=\int_{\partial \Omega}\langle\Theta(x, y), i \sigma(D ; \nu)(y) f(y)\rangle_{y} d S(y), \quad x \in \Omega, \\
& C_{D} f(x):=\text { p.v. } \int_{\partial \Omega}\langle\Theta(x, y), i \sigma(D ; \nu)(y) f(y)\rangle_{y} d S(y), \quad x \in \partial \Omega .
\end{aligned}
$$

The point is that $\mathcal{C}_{D}$ satisfies conditions similar to (2.9), (2.10) and (2.11). As a consequence, we have

$$
\operatorname{Im}\left(\frac{1}{2} I+C_{D} ; L^{p}(\Sigma, \mathcal{E})\right)=\mathcal{H}_{+}^{p}(\Sigma, D)=\operatorname{Ker}\left(-\frac{1}{2} I+C_{D} ; L^{p}(\Sigma, \mathcal{E})\right),
$$

for any $1<p<\infty$. One final property we want to single out is that the adjoint of $C_{D}$ acting on $L^{p}(\Sigma, \mathcal{E})$ is

$$
\left(C_{D}\right)^{*}=-i \sigma\left(D^{*} ; \nu\right) C_{D^{*}}\left[i \sigma\left(D^{*} ; \nu\right)\right]^{-1} \quad \text { on } L^{q}(\Sigma, \mathcal{E}), 1 / p+1 / q=1 .
$$

Here $C_{D^{*}}$ is the singular integral operator constructed analogously to $C_{D}$ but with $D^{*}$ replacing $D$. In particular, (2.24) remains valid with $D$ replaced by $D^{*}$. Thus, from (2.24) and the second equality in (2.23), we have

$$
\begin{aligned}
\operatorname{Ker} & \left(\frac{1}{2} I+\left(C_{D}\right)^{*} ; L^{p}(\Sigma, \mathcal{E})\right) \\
& =\operatorname{Ker}\left(\frac{1}{2} I-i \sigma\left(D^{*} ; \nu\right) C_{D^{*}}\left[i \sigma\left(D^{*} ; \nu\right)\right]^{-1} ; L^{p}(\Sigma, \mathcal{E})\right) \\
& =i \sigma\left(D^{*} ; \nu\right) \operatorname{Ker}\left(\frac{1}{2} I-C_{D^{*}} ; L^{p}(\Sigma, \mathcal{E})\right) \\
& =i \sigma\left(D^{*} ; \nu\right) \mathcal{H}_{+}^{p}\left(\Sigma, D^{*}\right) .
\end{aligned}
$$

Returning to the study of $\Phi$ in (2.18), we may write

$$
\begin{aligned}
\operatorname{Im} \Phi & =i \sigma\left(D^{*} ; \nu\right) \mathcal{H}_{+}^{p}\left(\Sigma, D^{*}\right)=\operatorname{Ker}\left(\left(\frac{1}{2} I+C_{D}\right)^{*} ; L^{p}(\Sigma, \mathcal{E})\right) \\
& =\left[\operatorname{Im}\left(\frac{1}{2} I+C_{D} ; L^{q}(\Sigma, \mathcal{E})\right)\right]^{\circ}=\left[\mathcal{H}_{+}^{q}(\Sigma, \mathcal{E})\right]^{\circ} .
\end{aligned}
$$

Hence $\Phi$ in (2.18) is onto also.

Step V. Here are the last details in the proof of (1.9)-(1.10). We only need to combine (2.15), (2.17) and the fact that the mapping (2.18) is an isomorphism in order to write

$$
\begin{aligned}
\operatorname{dim} \operatorname{Ker}\left(D^{*} ; L^{q}(\mathcal{M}, \mathcal{F})\right) & =\operatorname{dim} \operatorname{Ker}\left(D^{*} ; H^{1, q}(\mathcal{M}, \mathcal{F})\right) \\
& =\operatorname{dim}\left(\mathcal{H}_{-}^{q}\left(\Sigma, D^{*}\right) \cap \mathcal{H}_{+}^{q}\left(\Sigma, D^{*}\right)\right) \\
& =\operatorname{dim}\left(\mathcal{H}_{-}^{p}(\Sigma, D)^{\circ} \cap \mathcal{H}_{+}^{p}(\Sigma, D)^{\circ}\right) \\
& =\operatorname{dim}\left(\mathcal{H}_{-}^{p}(\Sigma, D)+\mathcal{H}_{+}^{p}(\Sigma, D)\right)^{\circ}
\end{aligned}
$$

where $1 / p+1 / q=1$. From this, the fact that $\mathcal{H}_{-}^{p}(\Sigma, D)+\mathcal{H}_{+}^{p}(\Sigma, D)$ is closed in $L^{p}(\Sigma, \mathcal{E})$ and $(2.17)$, the index formula (1.9) follows.

Finally, (1.10) is seen from (2.17) and (2.27). This completes the proof of the theorem. 


\section{REFERENCES}

[Ar] N. Aronszajn, A unique continuation theorem for solutions of elliptic differential equations or inequalities of second order, Journ. de Math. 36 (1957), 235-249. MR 19:1056c

[Bo] B. Bojarski, The abstract linear conjugation problem and Fredholm pairs of subspaces, In Memoriam I. N. Vekua, Tbilisi University, Tbilisi, 1979, 45-60. MR 83d:47021

[BBW1] B. Booß-Bavnbek and K. P. Wojciechowski, Desuspension of splitting elliptic symbols II, Ann. Global. Anal. Geom. 4 (1986), 349-400. MR 89f:58126

[BBW2] B. Booß-Bavnbek and K. P. Wojciechowski, Elliptic Boundary Problems for Dirac Operators, Birkhäuser, Boston-Basel-Berlin, 1993. MR 94h:58168

[CMcM] R. Coifman, A. McIntosh and Y. Meyer, L'intégrale de Cauchy définit un opérateur borné sur $L^{2}$ pour les courbes Lipschitziennes, Ann. of Math. 116 (1982), 361-387. MR 84m:42027

[Co] H. O. Cordes, Über die eindeutige Bestimmtheit der Lösungen elliptischer Differentialgleichungen durch Anfangsvorgaben, Nachr. Akad. Wiss. Göttingen Math.-Phys. Kl. 11 (1956), 239-258. MR 19:148a

[Da] G. David, Opérateurs intégraux singuliers sur certaines courbes du plan complexe, Ann. Sci. E.N.S. 17 (1984), 157-189. MR 85k:42026

[GM] J. Gilbert and M. A. Murray, Clifford Algebras and Dirac Operators in Harmonic Analysis, Cambridge Studies in Advanced Mathematics, Vol. 26, 1991. MR 93e:42027

[Ka] T. Kato, Perturbation Theory for Linear Operators, Springer, Berlin, $1976 . \quad$ MR 53:11389

[Ke] C. E. Kenig, Weighted $H^{p}$ spaces on Lipschitz domains, Amer. J. Math. 102 (1980), 129-163. MR 81d:30060

[LMcS] C. Li, A. McIntosh and S. Semmes, Convolution singular integrals on Lipschitz surfaces, J. Amer. Math. Soc. 5 (1992), 455-481. MR 93b:42029

[MeCo] Y. Meyer and R. R. Coifman, Ondelettes et opérateurs, Vol. III, Hermann, editeurs des sciences et des arts, Paris, 1990. MR 93i:42004

[Mi] M. Mitrea, Clifford Wavelets, Singular Integrals, and Hardy Spaces, Lecture Notes in Mathematics No. 1575, Springer-Verlag, Berlin, New York, 1994. MR 96e:31005

[Mi2] M. Mitrea, Generalized Dirac operators on nonsmooth manifolds and Maxwell's equations, in prepartion (1998).

[Mu N. I. Mushelišvili, Singular Integral Equations, English translation of the 1946 edition: Noordhoff, Groningen, 1953. MR 15:434e

[Ni] L. Nicolaescu, The Maslov index, the spectral flow, and decompositions of manifolds, Duke Math. J. 80 (1995), 485-533. MR 96k:58208

[Ta] M. E. Taylor, Pseudodifferential Operators and Nonlinear PDE, Birkhäuser, Boston, 1991. MR 92j:35193

Department of Mathematics, University of Missouri-Columbia, Columbia, MO 65211

E-mail address: marius@math.missouri.edu 